\title{
Optical parametric amplification in silicon nitride waveguides for coherent Raman imaging
}

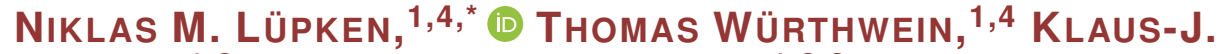 \\ BOLler, ${ }^{1,2}$ (D) AND CARSten FALLNICH ${ }^{1,2,3}$ (D) \\ ${ }^{1}$ Institute of Applied Physics, University of Münster, Corrensstraße 2, 48149 Münster, Germany \\ ${ }^{2}$ MESA + Institute for Nanotechnology, University of Twente, Enschede 7500 AE, The Netherlands \\ ${ }^{3}$ Cells in Motion Interfaculty Centre, University of Münster, Waldeyerstraße 15, 48149 Münster, Germany \\ ${ }^{4}$ These authors contributed equally to this work \\ *n.luepken@uni-muenster.de
}

\begin{abstract}
We present tunable waveguide-based optical parametric amplification by four-wave mixing (FWM) in silicon nitride waveguides, with the potential to be set up as an all-integrated device, for narrowband coherent anti-Stokes Raman scattering (CARS) imaging. Signal and idler pulses are generated via FWM with only $3 \mathrm{~nJ}$ pump pulse energy and stimulated by using only $4 \mathrm{~mW}$ of a continuous-wave seed source, resulting in a $35 \mathrm{~dB}$ enhancement of the idler spectral power density in comparison to spontaneous FWM. By using waveguides with different widths and tuning the wavelength of the signal wave seed, idler wavelengths covering the spectral region from $1.1 \mu \mathrm{m}$ up to $1.6 \mu \mathrm{m}$ can be generated. The versatility of the chip-based FWM light source is demonstrated by acquiring CARS images.
\end{abstract}

(C) 2021 Optical Society of America under the terms of the OSA Open Access Publishing Agreement

\section{Introduction}

Laser-based spectroscopy and imaging have become essential techniques for biochemical and medical applications, especially coherent anti-Stokes Raman scattering (CARS) microscopy [1]. A CARS signal is generated by the coherent excitation of a molecular resonance with two optical waves at different carrier frequencies. The benefits are chemically selectivity and a high signal yield, in comparison to spontaneous Raman scattering [2]. To generate a CARS signal the frequency difference of the two optical waves has to match the vibrational resonance frequency, which lies either in the fingerprint region $\left(<1800 \mathrm{~cm}^{-1}\right)$ containing all molecular-specific vibrations, the silent region $\left(1800 \mathrm{~cm}^{-1}-2800 \mathrm{~cm}^{-1}\right)$ including Raman tags and deuterated substances, or the $\mathrm{CH}$-stretch region $\left(2800 \mathrm{~cm}^{-1}-3200 \mathrm{~cm}^{-1}\right)$ with lipids and proteins [1]. Due to their wide wavelength range light sources most beneficial for the generation of CARS signals are optical parametric oscillators (OPOs), but they are critical to align and maintain as well as typically expensive and large [3].

In order to overcome the downsides of OPOs, cheaper and more robust fiber-based systems with a dual-color output for CARS applications have been developed. These systems, e.g. fiber-based optical parametric oscillators [4-7] and amplifiers [8,9] as well as spontaneous FWM-based light sources $[10,11]$ or supercontinuum sources $[12,13]$, exploit nonlinear frequency conversion in photonic crystal fibers (PCFs). However, due to the relatively low nonlinear refractive index coefficient of PCFs, high pulse energies are required. This renders it difficult to further reduce the size of the frequency conversion system, prohibiting an integration for on-chip Raman applications, i.e. robust biomedical system that are down-scaled to the size of a chip [14,15].

In contrast to fibers, silicon nitride $\left(\mathrm{Si}_{3} \mathrm{~N}_{4}\right)$ waveguides offer a versatile, CMOS-compatible, onchip platform with a high nonlinear refractive index and a tight mode confinement [16]. Therefore, nonlinear processes such as FWM can be driven more efficiently [17-21], enabling operation with lower pump energies and shorter interaction lengths. Frequency conversion by spontaneous FWM was already demonstrated in $\mathrm{Si}_{3} \mathrm{~N}_{4}$ for telecommunications [22], mid-infrared generation 
[23], and even for broadband CARS applications [24]. Furthermore, frequency conversion was already achieved with millimeter-scale waveguide lengths and pump energies down to the picojoule regime [23]. However, as spontaneous FWM starts from vacuum fluctuations, the conversion efficiencies are very low, in the order of $-20 \mathrm{~dB}$ to $-30 \mathrm{~dB}$ [22-24].

Stimulated FWM, i.e. seeding the parametric amplification process, results in a by orders of magnitude increased conversion efficiency, which was demonstrated in many different fiber-based optical parametric amplifiers [5,7,9]. Moreover, a waveguide-based optical parametric amplifier (WOPA) was demonstrated [23], however, the frequency conversion was used for mid-infrared generation which is unsuitable for coherent Raman imaging. A WOPA light source for Raman applications was demonstrated numerically but was not experimentally realized up to now [25].

Here, we present a WOPA light source based on stimulated FWM in $\mathrm{Si}_{3} \mathrm{~N}_{4}$ waveguides for narrowband CARS imaging. The dependence of the WOPA on the pump energy, pump pulse duration, seed power, seed wavelength, and waveguide dimensions is investigated in detail. Stimulating the FWM process with a continuous wave (cw) seed beam led to an enhancement of up to $35 \mathrm{~dB}$ compared to radiation generated by spontaneous FWM [24]. The increased output power enabled chemically selective CARS imaging covering vibrational resonances from the fingerprint to the $\mathrm{CH}$-stretch region. By acquiring CARS images we showed the versatility of the WOPA light source for laser-scanning microscopy.

\section{Setup of the waveguide optical parametric amplifier}

In order to set up a WOPA light source for narrowband CARS imaging, a narrowband pump beam and a narrowband Stokes beam, the latter with a higher wavelength than the pump wavelength, are necessary. Therefore, a pulsed narrowband pump beam and a narrowband $\mathrm{cw}$ seed beam are required to generate a narrowband idler sideband by stimulated FWM. A schematic of the input and output spectra is shown in Figs. 1(a) and (b), respectively. For CARS imaging, the wavelength of the cw seed is tuned such that its frequency difference to the pump equals the vibrational resonance of interest. The residual pump beam and the generated idler beam are then directed into the microscope.

(a) Input

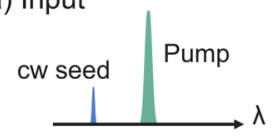

(b) Stim. FWM output

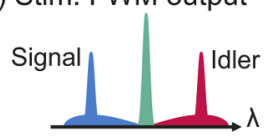

(c)

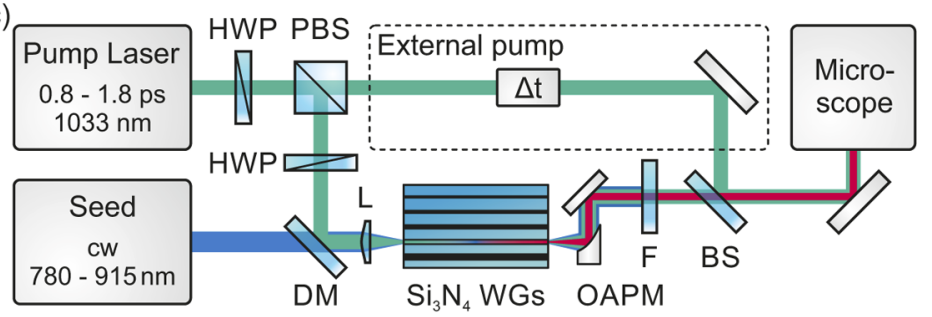

Fig. 1. Schematics of the spectral components of (a) the input and (b) the stimulated FWM output on a spontaneous FWM background of the waveguide (not to scale). (c) Schematic setup of CARS measurements driven by a FWM light source. For details see text.

The experimental setup is shown in Fig. 1(c): An ytterbium-doped fiber laser (Amplitude Systemes, Satsuma) emitted pulses centered at $1033 \mathrm{~nm}$ wavelength with a repetition rate of $1 \mathrm{MHz}$. As the FWM process does not depend on the repetition rate, we denote the used pulse energies in the following. In order to investigate the influence of the spectral bandwidth of the pump pulses, a Fourier filter (not shown) behind the pump laser was used to adjust the pump pulse duration between $0.8 \mathrm{ps}$ and $1.8 \mathrm{ps}$. Then, the pump pulses were split into two copies with a half-wave plate (HWP) and a polarizing beam-splitter (PBS). One pulse copy was launched in the TE-polarization (adjusted by a second HWP) together with a tunable continuous-wave (cw) seed with about $0.2 \mathrm{~nm}$ bandwidth from a titanium:sapphire (Ti:Sa, Schwartz Electro-Optics, 
Titan CW) laser (Fig. 1(a)) into a $7 \mathrm{~mm}$ long $\mathrm{Si}_{3} \mathrm{~N}_{4}$ waveguide (provided by LioniX International B.V.) using a dichroic mirror (DM, short-pass $950 \mathrm{~nm}$ ) and an aspherical lens (L, $3.1 \mathrm{~mm})$ in order to pump and stimulate the FWM process, respectively. The Ti:Sa laser system was used for a proof-of-concept and can be replaced by a tunable laser diode, integrated on the chip [26]. $\mathrm{Si}_{3} \mathrm{~N}_{4}$ waveguides with a fixed height of $950 \mathrm{~nm}$ and different widths ranging from $600 \mathrm{~nm}$ to $1500 \mathrm{~nm}$ in steps of $50 \mathrm{~nm}$ were available on a single chip. These waveguides are multi-mode at the considered wavelengths, however, the higher-order mode content was negligible when maximizing the coupling efficiency. The input coupling efficiencies of the pump and the seed beam were $35-40 \%$, depending on the waveguide width, and about $20 \%$, respectively. In the $\mathrm{Si}_{3} \mathrm{~N}_{4}$ waveguides, signal and idler sidebands were generated via stimulated FWM (Fig. 1(b)). In order to minimize a temporal walk-off and a spatial chirp of the output pulses an off-axis parabolic mirror (OAPM, $6.35 \mathrm{~mm}$ focal length) was used for collimation, and a subsequent long-pass filter (F, long-pass $1000 \mathrm{~nm}$ ) removed the signal sideband for the CARS experiments. The second pulse copy was bypassed around the waveguide and served as an external pump beam being overlapped spatially at a 70:30 beam splitter (BS, $70 \%$ transmission) and temporally by means of a delay line $(\Delta t)$ with the light emerging from the waveguide. Both beams were steered into a home-built microscope for narrowband CARS imaging (for details see section 4.).

\section{Waveguide-based optical parametric amplification}

In the following, the optical parametric amplification process is investigated by comparing spontaneous and stimulated FWM spectra. Furthermore, the wavelength tuning capability of the system is examined by measuring stimulated FWM spectra in waveguides with different widths, with different wavelengths and powers. Moreover, the influence of the pump pulse duration on the generated bandwidth is studied. Based on these investigations the optimal waveguide and pump pulse parameters depending on the application can be estimated for the WOPA light source.

\subsection{Spontaneous vs. stimulated four-wave mixing}

In order to compare spectra generated by spontaneous and stimulated FWM, the pump pulses were coupled into a $1400 \mathrm{~nm}$ wide $\mathrm{Si}_{3} \mathrm{~N}_{4}$ waveguide, with and without the cw seed laser beam stimulating the FWM process. The output spectra were measured with an optical spectrum analyzer (OSA, Yokogawa, AQ-6370D) for a pump pulse duration of $0.8 \mathrm{ps}$ and an input energy of $3 \mathrm{~nJ}$, which was well below the damage threshold of the waveguides of about $6 \mathrm{~nJ}$. The seed laser was set to $857 \mathrm{~nm}$ wavelength for an exemplary measurement with waveguide internal powers ranging from $0 \mathrm{~mW}$ to $4 \mathrm{~mW}$. The spontaneous FWM process resulted in broadband signal and idler sidebands around the pump (red curve in Fig. 2(a)), extending from $830 \mathrm{~nm}$ to $970 \mathrm{~nm}$ and from $1100 \mathrm{~nm}$ to $1350 \mathrm{~nm}$, respectively. The broadband signal and idler sidebands were in agreement with the phase-matching condition and are suitable for broadband CARS spectroscopy [24]. In contrast to the broadband spontaneous FWM sidebands, two narrowband peaks emerge in the stimulated case at $857 \mathrm{~nm}$ and $1300 \mathrm{~nm}$ (blue curve in Fig. 2(a)), when the pump pulses, spectrally broadening by self-phase modulation (SPM) in the waveguide, were launched together with the seed beam into the waveguide. The narrowband seed is observed on top of the stimulated signal sideband at $857 \mathrm{~nm}$ wavelength. Note that the seed bandwidth of $0.2 \mathrm{~nm}$ appears broader on account of the $2 \mathrm{~nm}$ resolution of the OSA. The spectral power density of the stimulated FWM sidebands increased for higher seed powers and reached an enhancement of $35 \mathrm{~dB}$, measured with $4 \mathrm{~mW}$ seed power in comparison to the spontaneous FWM sideband. In a similar experiment, $25 \mathrm{~dB}$ enhancement was achieved in $1 \mathrm{~cm}$ long $\mathrm{Si}_{3} \mathrm{~N}_{4}$ waveguides [23]. In contrast, up to $50 \mathrm{~dB}$ enhancement was reached in PCFs [8,9], however, when taking the used fiber lengths and peak powers into account, a reduction of approximately two orders of magnitude in both, necessary peak power and waveguide length, was accomplished in comparison to fiber experiments. 

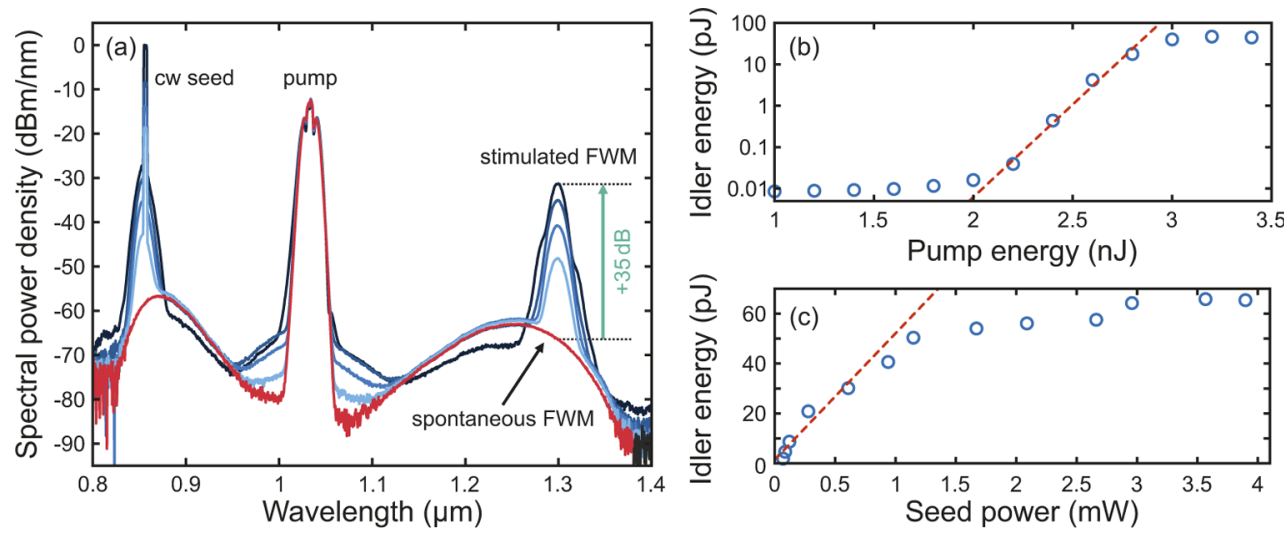

Fig. 2. (a) Spectra of spontaneous (red) and stimulated (blue) FWM, the latter seeded at $857 \mathrm{~nm}$ wavelength, in a $1400 \mathrm{~nm}$ wide $\mathrm{Si}_{3} \mathrm{~N}_{4}$ waveguide. The pump energy was set to $3 \mathrm{~nJ}$. Idler energy (blue circles) as a function of (b) the pump energy (note the logarithmic scale, $4 \mathrm{~mW}$ seed power) and (c) of the seed power ( $3 \mathrm{~nJ}$ pump energy). The red dashed lines show an exponential and a linear fit, respectively.

The output energy of the spontaneous FWM idler sideband was $0.25 \mathrm{pJ}$, integrated across the spectral range from $1.1 \mu \mathrm{m}$ to $1.4 \mu \mathrm{m}$. In the stimulated case, the output energy of the idler band reached approximately $65 \mathrm{pJ}$ (integrated over the same bandwidth). Thus, the achieved external conversion efficiency corresponded to $-16.6 \mathrm{~dB}$. This value represents a much higher conversion efficiency per medium length in comparison to an efficiency of $-9.8 \mathrm{~dB}$ in a $30 \mathrm{~cm}$ long PCF [8] and $-27 \mathrm{~dB}$ in a $1 \mathrm{~cm}$ long $\mathrm{Si}_{3} \mathrm{~N}_{4}$ waveguide [23].

Figure 2(b) shows the idler energy as a function of the pump energy revealing an exponential relation, which is in agreement with theory [27]. For pump energies below $1.8 \mathrm{~nJ}$ the power spectral density of the FWM sidebands was lower than the noise floor of the OSA. For pump energies higher than $3 \mathrm{~nJ}$ the output idler energy saturated. The saturation originates from back conversion [9], which was verified by accompanying numerical simulations. Moreover, a linear relationship was observed between the waveguide internal seed power and the idler output energy as depicted in Fig. 2(c) until saturation occurred. Already $86 \%$ of the maximum output energy was reached for a seed power of only $2 \mathrm{~mW}$. For even higher seed powers the output energy increased slightly, however, the spectral shape got distorted (compare Fig. 2(a)). With numerical simulations this distortion could be attributed to SPM. Furthermore, numerical simulations showed that further increasing the seed power would result in reduced output idler energy due to back conversion, a well-known effect within parametric amplification [9].

\subsection{Wavelength tuning via waveguide dispersion and seed wavelength}

The parametric gain for $\mathrm{FWM}$ in $\mathrm{Si}_{3} \mathrm{~N}_{4}$ waveguides is determined by the dispersion profile and, therefore, can be engineered via the cross-sectional geometry of the waveguide [17,19,24]. The parametric FWM gain broadens, but decreases for wider waveguides, until a waveguide width is reached where the pump lies in the normal dispersion regime, which was already numerically shown in a previous study [24]. Within the gain region, seeding leads to parametric amplification by stimulated FWM. Therefore, wavelength tuning can be accomplished by tuning the seed wavelength. The gain region, in which the seed can be tuned, is determined by using different waveguide widths and, therefore, different dispersion profiles.

In order to investigate the dependence of the parametric gain on the waveguide dimensions, measurements in different waveguides with $3 \mathrm{~nJ}$ input energy and $0.8 \mathrm{ps}$ pump pulse duration were performed. The experimental results of the dispersion-engineered spontaneous FWM spectra are 
shown in Fig. 3(a) measured in $\mathrm{Si}_{3} \mathrm{~N}_{4}$ waveguides with widths $w$ of $1300 \mathrm{~nm}$ (blue), $1400 \mathrm{~nm}$ (red), and $1450 \mathrm{~nm}$ (green), respectively. All spectra showed broadband signal and idler sidebands around the residual SPM-broadened pump. Additionally, a cascaded FWM sideband was visible in the $1300 \mathrm{~nm}$ wide waveguide around $780 \mathrm{~nm}$, which was about $15 \mathrm{~dB}$ weaker than the signal sideband around $880 \mathrm{~nm}$. The measurements agreed with the calculations of the parametric FWM gain from Ref. [24]. The use of all three waveguides with their different widths enabled the realization of a light source covering the wavelength region from approximately $750 \mathrm{~nm}$ up to $1600 \mathrm{~nm}$. For CARS experiments, this wavelength range allows to address the complete Raman spectrum from the fingerprint region $\left(<1800 \mathrm{~cm}^{-1}\right.$, blue shaded area) containing all molecular specific vibrations, over the silent region $\left(1800 \mathrm{~cm}^{-1}-2800 \mathrm{~cm}^{-1}\right.$, red shaded area) including Raman tags and deuterated substances, to the $\mathrm{CH}$-stretch region $\left(2800 \mathrm{~cm}^{-1}-3200 \mathrm{~cm}^{-1}\right.$, green shaded area) wherein, e.g., lipids and proteins show a Raman response.
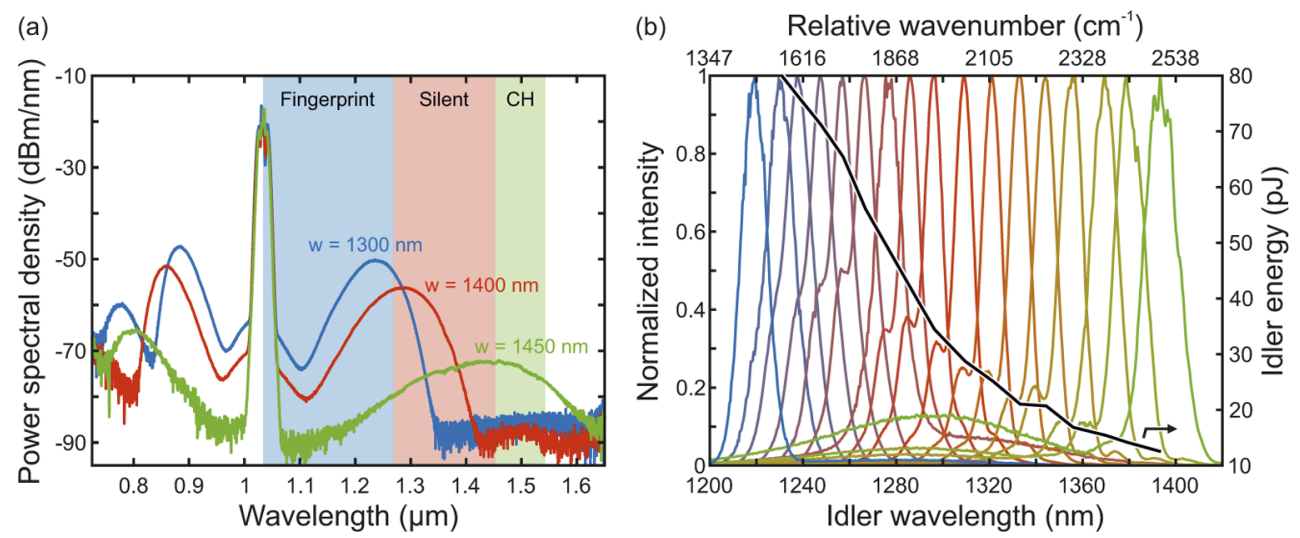

Fig. 3. (a) Spontaneous FWM spectra measured with $3 \mathrm{~nJ}$ pump energy in waveguides with a height of $950 \mathrm{~nm}$ and widths $w$ of $1300 \mathrm{~nm}$ (blue), $1400 \mathrm{~nm}$ (red), and $1450 \mathrm{~nm}$ (green). The shaded areas mark the fingerprint $\left(<1800 \mathrm{~cm}^{-1}\right.$, blue $)$, the silent $\left(1800 \mathrm{~cm}^{-1}-2800 \mathrm{~cm}^{-1}\right.$, red), and the $\mathrm{CH}$-stretch region $\left(2800 \mathrm{~cm}^{-1}-3200 \mathrm{~cm}^{-1}\right.$, green). (b) Idler spectra of stimulated FWM on a linear scale and normalized to the corresponding idler energy (black curve), measured in the $1400 \mathrm{~nm}$ wide waveguide with seed wavelengths tuned between $820 \mathrm{~nm}$ and $895 \mathrm{~nm}$.

In future experiments, re-alignment of the setup related to changing waveguides could be avoided by using a wavelength-tunable pump oscillator. Numerical calculations showed that on account of the phase-matching condition only small pump wavelength changes in the order of a few tens of nanometers would be sufficient to cover all three above-listed wavenumber regions, similar to experiments in PCFs [7]. Alternatively, engineering the dispersion profile along the propagation direction by specific changes of the waveguide width and height [28], already applied to nonlinear pulse compression and supercontinuum generation [29,30], could allow for an ultra-broadband FWM spectrum covering all three wavenumber regions of interest (compare Fig. 3(a)) in a single waveguide.

Beside the coarse wavelength range selection by choosing different waveguide widths, the seeding process greatly enhanced the FWM conversion efficiency in a specific wavelength range and enabled a fine tuning by adjusting the seed wavelength. As the seed laser was operated in continuous-wave mode, no changes within the setup, such as delay lines for keeping temporal overlap, were necessary when tuning the seed wavelength. In order to characterize the wavelength fine tuning capabilities of the stimulated FWM process, output spectra of the $1400 \mathrm{~nm}$ wide $\mathrm{Si}_{3} \mathrm{~N}_{4}$ waveguide were acquired for different seed wavelengths ranging from $820 \mathrm{~nm}$ to $895 \mathrm{~nm}$ (see Fig. 3(b)). The output spectra, shown in Fig. 3(b), were individually normalized corresponding to 
the pulse energy (black curve in Fig. 3(b)). The idler energy decreases for longer wavelengths due to the reduced parametric gain (compare Fig. 3(a)) and the tuning on the short wavelength side was limited by the used seed laser. Using this single waveguide a spectral range of approximately $180 \mathrm{~nm}$ was covered, which corresponds to approximately $1477 \mathrm{~cm}^{-1}$ to $2512 \mathrm{~cm}^{-1}$, addressing the spectral region of Raman tags and deuterated samples.

\subsection{Power and bandwidth dependencies for different pump pulse durations}

In CARS spectroscopy, the broadest bandwidth of the involved pulses determines the spectral resolution. In our experiment, the idler bandwidth was determined by the pump bandwidth, i.e. the pump bandwidth limited the spectral resolution. However, reducing the spectral bandwidth leads to a reduced peak power at a fixed pulse energy resulting in a reduced CARS signal. Therefore, a compromise between signal strength and spectral resolution has to be made.

In order to investigate the influence of the spectral pump bandwidth on the idler sideband generation, the duration of the pump pulses was varied with a spectral Fourier filter from $0.8 \mathrm{ps}$ to $1.8 \mathrm{ps}$ before coupling into the $\mathrm{Si}_{3} \mathrm{~N}_{4}$ waveguides. This results in a bandwidth reduction of the pump pulses from $3.9 \mathrm{~nm}\left(36.4 \mathrm{~cm}^{-1}\right)$ for a duration of $0.8 \mathrm{ps}$ down to $1.4 \mathrm{~nm}\left(13.4 \mathrm{~cm}^{-1}\right)$ for $1.8 \mathrm{ps}$. The stimulated FWM spectra were measured with an exemplary seed wavelength of $900 \mathrm{~nm}$ in a waveguide with a width of $1300 \mathrm{~nm}$, providing the highest gain. The stimulated FWM signal, shown in Fig. 4(a) for a fixed pump pulse energy of $3 \mathrm{~nJ}$, increased and broadened for shorter pump pulse durations. This observation can be explained by the higher peak power for shorter pulse durations and, accordingly, broader pump pulse spectra at fixed pulse energy. We limited the pulse energy to stay well below the experimentally determined (pulse duration independent) damage threshold of $6 \mathrm{~nJ}$, such that the maximum peak power was limited for longer pulse durations. The integrated idler output power (from $1.1 \mu \mathrm{m}$ to $1.3 \mu \mathrm{m}$ ), plotted in Fig. 4(b) as circles, decreased exponentially by more than $40 \mathrm{~dB}$ at $1.8 \mathrm{ps}$ pulse duration in comparison to $0.8 \mathrm{ps}$. This exponential dependence of the idler output power was addressed to the measured exponential dependence on the peak power (compare Fig. 2(b)), which scales linearly with the pulse duration.
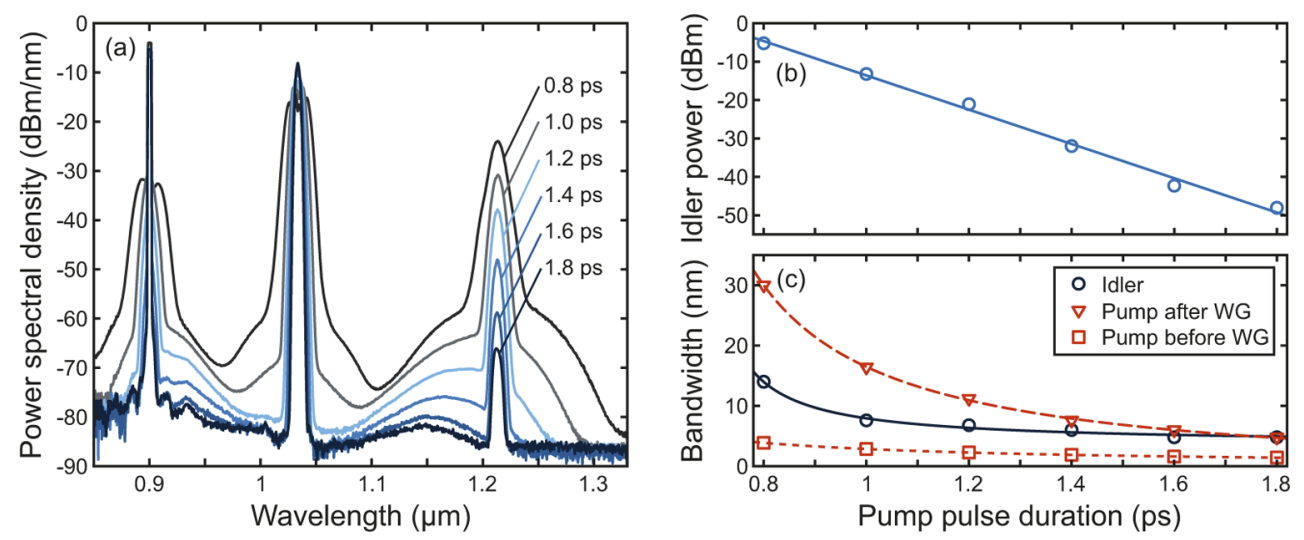

Fig. 4. (a) Output spectra of the $1300 \mathrm{~nm}$ wide waveguide for pump pulse durations in the range from $0.8 \mathrm{ps}$ (gray) to $1.8 \mathrm{ps}$ (dark blue). (b) Idler output power as a function of the pump pulse duration. A linear fit was added to guide the eye. (c) Bandwidth of the idler pulses (circles) as well as of the pump pulses before (squares) and after (triangles) the waveguide are plotted as a function of the pump pulse duration. Hyperbola fits were added to guide the eye.

The measured full width at half maximum spectral bandwidths of the idler pulses (circles) as well as of the pump pulses before (squares) and after (triangles) the waveguide are shown 
for the investigated pump pulse durations in Fig. 4(c). The measured spectral bandwidth values followed a hyperbolic function of the pump pulse duration, which can be expected from the time-bandwidth product. The bandwidth of the pump pulses was larger after the waveguide on account of spectral broadening by SPM (see Fig. 4(a)). The pump spectra broadened by a factor of 7.7 for $0.8 \mathrm{ps}$ pulse duration and a factor of 3.4 for $1.8 \mathrm{ps}$, due to the reduced peak power for longer pulses. With increasing pump pulse duration, the idler bandwidth decreased accordingly from $14 \mathrm{~nm}\left(95.2 \mathrm{~cm}^{-1}\right)$ to $4.8 \mathrm{~nm}\left(36.2 \mathrm{~cm}^{-1}\right)$. With these bandwidths CARS imaging of single resonances can be accomplished as demonstrated in the subsequent section. However, if required, the spectral resolution for CARS can be further improved by well-known techniques such as, e.g., spectral focusing [31], which would only require a longer waveguide to induce a frequency chirp via dispersion.

\section{Chemically selective CARS imaging}

Using stimulated FWM in $\mathrm{Si}_{3} \mathrm{~N}_{4}$ waveguides for chemically selective CARS imaging applications has advantages in comparison to spontaneous FWM for broadband CARS spectroscopy measurements [24], as the stimulated idler band has a higher power spectral density and can be easily tuned in wavelength via the seed radiation. Moreover, an advantage when using a narrowband pump for the CARS measurements is that a single pixel detector, for instance a photomultiplier tube (PMT), can be applied, resulting in a significantly lower pixel dwell time in the $\mu \mathrm{s}$-regime in comparison to the ms-regime when using a spectrometer for broadband CARS. All of these advantages enable fast acquisition times suitable for narrowband CARS imaging.

For the CARS experiments a home-built laser-scanning microscope was used (see Fig. 5(a)), which consisted of a galvo-scanner pair (GS, Sunny Technology, TSH8203), a scan and a tube lens (SL and TL, both spherical $f=60 \mathrm{~mm}$ lenses), a focusing microscope objective (MO, $\mathrm{NA}=0.6)$, and a second MO $(\mathrm{NA}=0.65)$ to collect the CARS signal generated in the sample (S). A dichroic mirror (DM, long-pass $900 \mathrm{~nm}$ ) and a set of filters (F, short-pass $900 \mathrm{~nm}$ and long-pass $750 \mathrm{~nm}$ ) directed the light onto a PMT (Hamamatsu, H7422-50), which detected the non-descanned forward-CARS signal. Lock-in detection (Zurich Instruments, HF2LI) at the laser repetition rate of $1 \mathrm{MHz}$ was used for a higher signal-to-background ratio. A photodiode (PD, Thorlabs, PDA100A2) detected the residual pump light, which was transmitted through the DM, to simultaneously acquire brightfield images of the sample. The total energy in the imaging plane was approximately $7.3 \mathrm{~nJ}$ of the external pump beam and approximately $30 \mathrm{pJ}$ of the idler beam, serving as the pump and Stokes waves for the CARS process, respectively. For highest signal-to-background ratio a pump pulse duration of $0.8 \mathrm{ps}$ was used in order to benefit from the high peak power taking a non-ideal spectral resolution into account.

The spectral resolution was determined by measuring a CARS spectrum around the isolated dimethyl sulfoxide- $\mathrm{d}_{6}$ (dDMSO) resonance in the silent region from $2020 \mathrm{~cm}^{-1}$ to $2210 \mathrm{~cm}^{-1}$ via tuning the seed wavelengths from $838 \mathrm{~nm}$ to $856 \mathrm{~nm}$, as dDMSO is of high interest for biomedical research [32]. In Fig. 5(b) the normalized CARS intensity, weighted with the wavelengthdependent idler energy, is shown as a function of the wavenumber, which was calculated from the idler's central wavelength. The spectral resolution, determined by a Lorentzian line fit (black curve in Fig. 5(b)), was approximately $85 \mathrm{~cm}^{-1}$ and corresponded to the spectral bandwidth of the idler pulses. Due to this spectral resolution the dispersive lineshape of the Raman resonance in CARS [33] is hardly resolved. The seed source could not be tuned to wavelengths corresponding to the wavenumbers around $2150 \mathrm{~cm}^{-1}$, which can be improved with a more reliable seed source. Nevertheless, the WOPA light source showed a high stability and tuning capability, which enabled the spectral measurement of the vibrational resonance. Furthermore, the spectral measurement of the resonance demonstrates the chemical selectivity, as the measured CARS intensity is reduced by more than $80 \%$ when measured off resonance, e.g., at $2020 \mathrm{~cm}^{-1}$, in comparison to a measurement on resonance, i.e., at $2125 \mathrm{~cm}^{-1}$. 


\section{Optics EXPRESS}

(a)

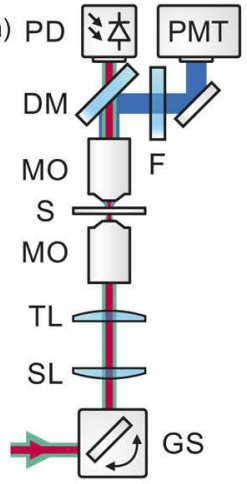

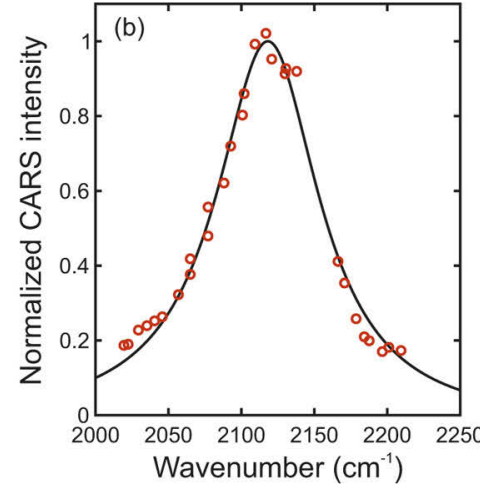

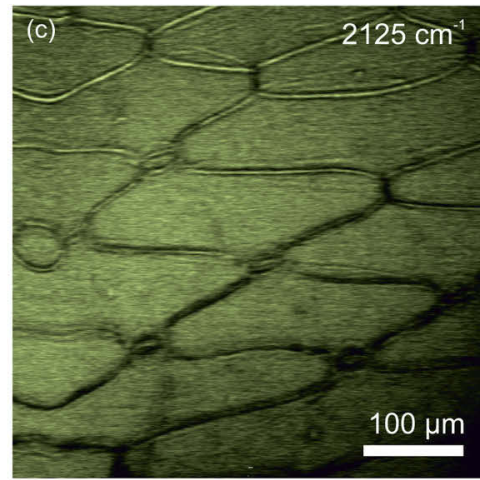

Fig. 5. (a) Schematic setup of the laser-scanning microscope. For abbreviations see text. (b) Normalized CARS intensity (red circles) as a function of the wavenumber measured across the resonance at $2125 \mathrm{~cm}^{-1}$ of dDMSO, with the black line representing a Lorentzian fit. (c) Forward-CARS image at $2125 \mathrm{~cm}^{-1}$ of a cross-sectional cut of a chlorophytum comosum leaf soaked with dDMSO. Three images with 1024 x 1024 pixels were acquired and averaged using a time constant of $10 \mu$ s per pixel.

To demonstrate the potential of the WOPA light source for imaging applications, CARS images of a cross-sectional cut of a leaf of chlorophytum comosum (known as airplane plant), soaked with dDMSO for immediate imaging, were acquired with the WOPA light source. The resonance of dDMSO at $2125 \mathrm{~cm}^{-1}$ was addressed by tuning the seed laser to $847 \mathrm{~nm}$. The intensity of the CARS signal is plotted in false colors in Fig. 5(c) (1024 x 1024 pixels, lock-in time constant $10 \mu \mathrm{s})$. The images were averaged three times for the best trade-off between the signal-to-noise ratio and the acquisition speed, such that the cell structure as well as the stomata could be visualized and identified clearly.

The lowest possible energy for imaging in the external pump beam was approximately $3 \mathrm{~nJ}$, when using a lock-in time constant of $10 \mu \mathrm{s}$. For external pump energies higher than $10 \mathrm{~nJ}$ it was possible to acquire images with a pixel dwell time of $1 \mu \mathrm{s}$, corresponding to only one pulse per pixel. Moreover, the spectral resolution can be enhanced by increasing the pump pulse duration (compare Sec. 3.3), but then the resulting lower idler power has to be compensated by higher external pump energies or longer pixel dwell times.

\section{Conclusion}

We presented a tunable light source, with the potential to be further integrated, based on stimulated four-wave mixing in silicon nitride waveguides. With a cw seed beam with $4 \mathrm{~mW}$ power and $3 \mathrm{~nJ}$ pump energy, $65 \mathrm{pJ}$ of idler energy were measured, corresponding to an enhancement of $35 \mathrm{~dB}$ compared to spontaneous FWM, thus, a conversion efficiency of $-16.6 \mathrm{~dB}$ was achieved. This conversion efficiency is about $10 \mathrm{~dB}$ higher as in comparable waveguide experiments [23], and in comparison to experiments in PCFs [8,9] the stimulated FWM results are improved by two orders of magnitude in medium length as well as in pulse power. Using different waveguide widths accomplished to pre-select the range of the FWM gain, and tuning the wavelength of the seed laser allowed for fine tuning the idler wavelength, ultimately covering the spectral range from $1.1 \mu \mathrm{m}$ to $1.6 \mu \mathrm{m}$. Within this spectral range the complete Raman spectrum from the fingerprint to the $\mathrm{CH}$-stretch region can be addressed using three waveguide widths, which was not possible with other waveguide-based light sources [22,23]. In CARS measurements, a spectral resolution of $85 \mathrm{~cm}^{-1}$ was estimated, but can be improved with longer pump pulse durations in combination 
with higher external pump energies or increased pixel dwell times. Nevertheless, CARS images of chlorophytum comosum soaked with dDMSO were acquired with a pixel dwell time of $10 \mu \mathrm{s}$.

In future experiments, the external seed source could be integrated on-chip to reduce the footprint of the WOPA scheme [34]. Furthermore, by integrating the samples on the chip, e.g. within microfluidic channels [35] or via evanescent field sensing [15], as well as the spectrometer for detecting the spectra [36], robust lab-on-a-chip CARS measurements could be enabled.

Acknowledgments. The authors thank Jörn P. Epping and LioniX International B.V. for support and fabrication of the silicon nitride waveguides.

Disclosures. The authors declare no conflicts of interest.

\section{References}

1. C. L. Evans and X. S. Xie, "Coherent Anti-Stokes Raman Scattering Microscopy: Chemical Imaging for Biology and Medicine," Annu. Rev. Anal. Chem. 1(1), 883-909 (2008).

2. L.-P. Choo-Smith, H. G. M. Edwards, H. P. Endtz, J. M. Kros, F. Heule, H. Barr, J. S. Robinson, H. A. Bruining, and G. J. Puppels, "Medical applications of Raman spectroscopy: From proof of principle to clinical implementation," Biopolymers 67(1), 1-9 (2002).

3. M. Jurna, J. P. Korterik, H. L. Offerhaus, and C. Otto, "Noncritical phase-matched lithium triborate optical parametric oscillator for high resolution coherent anti-Stokes Raman scattering spectroscopy and microscopy," Appl. Phys. Lett. 89(25), 251116 (2006).

4. J. E. Sharping, "Microstructure Fiber Based Optical Parametric Oscillators," J. Lightwave Technol. 26(14), 2184-2191 (2008).

5. M. Brinkmann, S. Janfrüchte, T. Hellwig, S. Dobner, and C. Fallnich, "Electronically and rapidly tunable fiberintegrable optical parametric oscillator for nonlinear microscopy," Opt. Lett. 41(10), 2193-2196 (2016).

6. K. Yang, S. Zheng, Y. Wu, P. Ye, K. Huang, Q. Hao, and H. Zeng, "Low-repetition-rate all-fiber integrated optical parametric oscillator for coherent anti-Stokes Raman spectroscopy," Opt. Express 26(13), 17519-17528 (2018).

7. M. Brinkmann, A. Fast, T. Hellwig, I. Pence, C. L. Evans, and C. Fallnich, "Portable all-fiber dual-output widely tunable light source for coherent Raman imaging," Biomed. Opt. Express 10(9), 4437-4449 (2019).

8. S. Lefrancois, D. Fu, G. R. Holtom, L. Kong, W. J. Wadsworth, P. Schneider, R. Herda, A. Zach, X. Sunney Xie, and F. W. Wise, "Fiber four-wave mixing source for coherent anti-Stokes Raman scattering microscopy," Opt. Lett. 37(10), 1652-1654 (2012).

9. M. Chemnitz, M. Baumgartl, T. Meyer, C. Jauregui, B. Dietzek, J. Popp, J. Limpert, and A. Tünnermann, "Widely tuneable fiber optical parametric amplifier for coherent anti-Stokes Raman scattering microscopy," Opt. Express 20(24), 26583-26595 (2012).

10. M. Baumgartl, M. Chemnitz, C. Jauregui, T. Meyer, B. Dietzek, J. Popp, J. Limpert, and A. Tünnermann, "All-fiber laser source for CARS microscopy based on fiber optical parametric frequency conversion," Opt. Express 20(4), 4484-4493 (2012)

11. M. Baumgartl, T. Gottschall, J. Abreu-Afonso, A. Díez, T. Meyer, B. Dietzek, M. Rothhardt, J. Popp, J. Limpert, and A. Tünnermann, "Alignment-free, all-spliced fiber laser source for CARS microscopy based on four-wave-mixing," Opt. Express 20(19), 21010-21018 (2012).

12. B. von Vacano and M. Motzkus, "Time-resolved two color single-beam CARS employing supercontinuum and femtosecond pulse shaping," Opt. Commun. 264(2), 488-493 (2006).

13. M. Okuno, H. Kano, P. Leproux, V. Couderc, and H.-o. Hamaguchi, "Ultrabroadband multiplex CARS microspectroscopy and imaging using a subnanosecond supercontinuum light source in the deep near infrared," Opt. Lett. 33(9), 923-925 (2008).

14. C. H. Camp Jr., S. Yegnanarayanan, A. A. Eftekhar, H. Sridhar, and A. Adibi, "Multiplex coherent anti-Stokes Raman scattering (MCARS) for chemically sensitive, label-free flow cytometry," Opt. Express 17(25), 22879-22889 (2009).

15. A. Dhakal, A. Z. Subramanian, P. Wuytens, F. Peyskens, N. L. Thomas, and R. Baets, "Evanescent excitation and collection of spontaneous Raman spectra using silicon nitride nanophotonic waveguides," Opt. Lett. 39(13), 4025-4028 (2014).

16. J. P. Epping, M. Hoekman, R. Mateman, A. Leinse, R. G. Heideman, A. van Rees, P. J. van der Slot, C. J. Lee, and K.-J. Boller, "High confinement, high yield Si_3N_4 waveguides for nonlinear optical applications," Opt. Express 23(2), 642-648 (2015).

17. J. P. Epping, T. Hellwig, M. Hoekman, R. Mateman, A. Leinse, R. G. Heideman, A. van Rees, P. J. van der Slot, C. J. Lee, C. Fallnich, and K.-J. Boller, "On-chip visible-to-infrared supercontinuum generation with more than $495 \mathrm{THz}$ spectral bandwidth," Opt. Express 23(15), 19596-19604 (2015).

18. K. Luke, Y. Okawachi, M. R. E. Lamont, A. L. Gaeta, and M. Lipson, "Broadband mid-infrared frequency comb generation in a Si3N4 microresonator," Opt. Lett. 40(21), 4823-4826 (2015).

19. M. A. G. Porcel, F. Schepers, J. P. Epping, T. Hellwig, M. Hoekman, R. G. Heideman, P. J. M. van der Slot, C. J. Lee, R. Schmidt, R. Bratschitsch, C. Fallnich, and K.-J. Boller, "Two-octave spanning supercontinuum generation in stoichiometric silicon nitride waveguides pumped at telecom wavelengths," Opt. Express 25(2), 1542-1554 (2017). 


\section{Optics EXPRESS}

20. N. M. Lüpken, T. Hellwig, M. Schnack, J. P. Epping, K.-J. Boller, and C. Fallnich, "Low-power broadband all-optical switching via intermodal cross-phase modulation in integrated optical waveguides," Opt. Lett. 43(8), 1631-1634 (2018).

21. A. L. Gaeta, M. Lipson, and T. J. Kippenberg, "Photonic-chip-based frequency combs," Nat. Photonics 13(3), 158-169 (2019).

22. J. S. Levy, A. Gondarenko, A. C. Turner-Foster, M. A. Foster, A. L. Gaeta, and M. Lipson, "Four-wave Mixing in Integrated Silicon Nitride Waveguides," in Conf. Lasers Electro-Optics/International Quantum Electron. Conf., (OSA, 2009), p. CMFF5.

23. A. S. Kowligy, D. D. Hickstein, A. Lind, D. R. Carlson, H. Timmers, N. Nader, D. L. Maser, D. Westly, K. Srinivasan, S. B. Papp, and S. A. Diddams, "Tunable mid-infrared generation via wide-band four-wave mixing in silicon nitride waveguides," Opt. Lett. 43(17), 4220-4223 (2018).

24. N. Lüpken, T. Würthwein, J. Epping, K. Boller, and C. Fallnich, "Spontaneous four-wave mixing in silicon nitride waveguides for broadband coherent anti-Stokes Raman scattering spectroscopy," Opt. Lett. 45(14), 3873-3876 (2020).

25. J. P. Epping, M. Kues, P. J. van der Slot, C. J. Lee, C. Fallnich, and K.-J. Boller, "Integrated CARS source based on seeded four-wave mixing in silicon nitride," Opt. Express 21(26), 32123-32129 (2013).

26. S. Kumari, J. Gustavsson, E. P. Haglund, J. Bengtsson, A. Larsson, G. Roelkens, and R. Baets, "Design of an 845-nm GaAs Vertical-Cavity Silicon-Integrated Laser with an Intracavity Grating for Coupling to a SiN Waveguide Circuit," IEEE Photonics J. 9(4), 1-9 (2017).

27. G. Agrawal, Nonlinear Fiber Optics (Academic Press, 2013).

28. P. Zhao, Z. Ye, K. Vijayan, C. Naveau, J. Schröder, M. Karlsson, and P. A. Andrekson, "Waveguide tapering for improved parametric amplification in integrated nonlinear Si 3 N 4 waveguides," Opt. Express 28(16), 23467-23477 (2020).

29. D. R. Carlson, P. Hutchison, D. D. Hickstein, and S. B. Papp, "Generating few-cycle pulses with integrated nonlinear photonics," Opt. Express 27(26), 37374-37382 (2019).

30. N. Singh, D. Vermulen, A. Ruocco, N. Li, E. Ippen, F. X. Kärtner, and M. R. Watts, "Supercontinuum generation in varying dispersion and birefringent silicon waveguide," Opt. Express 27(22), 31698-31712 (2019).

31. T. Hellerer, A. M. Enejder, and A. Zumbusch, "Spectral focusing: High spectral resolution spectroscopy with broad-bandwidth laser pulses," Appl. Phys. Lett. 85(1), 25-27 (2004).

32. S. Bégin, B. Burgoyne, V. Mercier, A. Villeneuve, R. Vallée, and D. Côté, "Coherent anti-Stokes Raman scattering hyperspectral tissue imaging with a wavelength-swept system,” Biomed. Opt. Express 2(5), 1296-1306 (2011).

33. H. Rigneault and P. Berto, "Tutorial: Coherent Raman light matter interaction processes," APL Photonics 3(9), 091101 (2018).

34. K.-J. Boller, A. van Rees, Y. Fan, J. Mak, R. E. M. Lammerink, C. A. A. Franken, P. J. M. van der Slot, D. A. I. Marpaung, C. Fallnich, J. P. Epping, R. M. Oldenbeuving, D. Geskus, R. Dekker, I. Visscher, R. Grootjans, C. G. H. Roeloffzen, M. Hoekman, E. J. Klein, A. Leinse, and R. G. Heideman, "Hybrid Integrated Semiconductor Lasers with Silicon Nitride Feedback Circuits," Photonics 7(1), 4-33 (2019).

35. K. Wörhoff, R. G. Heideman, A. Leinse, and M. Hoekman, "TriPleX: a versatile dielectric photonic platform," Adv. Opt. Technol. 4(2), 189-207 (2015).

36. X. Nie, E. Ryckeboer, G. Roelkens, and R. Baets, "CMOS-compatible broadband co-propagative stationary Fourier transform spectrometer integrated on a silicon nitride photonics platform,” Opt. Express 25(8), A409-A418 (2017) 